\title{
Radiative processes as a condensation phenomenon and the physical meaning of deformed canonical structures
}

\author{
J. Gamboa* \\ Departamento de Física, Universidad de Santiago de Chile, Casilla 307, Santiago, Chile \\ L. S. Grigorid \\ Instituto de Física, Universidade Federal do Rio de Janeiro, 21945, Rio de Janeiro, Brazil \\ M. S. Guimaraed $\ddagger$ \\ Instituto de Física, Universidade Federal do Rio de Janeiro, 21945, Rio de Janeiro, Brazil \\ F. Méndez§ \\ Departamento de Fúsica, Universidad de Santiago de Chile, Casilla 30\%, Santiago, Chile \\ C. Wotzasek \\ Instituto de Física, Universidade Federal do Rio de Janeiro, 21945, Rio de Janeiro, Brazil
}

(Dated: October 30, 2018)

\begin{abstract}
Working with well known models in $(2+1) D$ we discuss the physics behind the deformation of the canonical structure of these theories. A new deformation is constructed linking the massless scalar field theory with the self-dual theory. This is the exact dual of the known deformation connecting the Maxwell theory with the Maxwell-Chern-Simons theory. Duality is used to establish a web of relations between the mentioned theories and a physical picture of the deformation procedure is suggested.
\end{abstract}

*Electronic address: jgamboa@usach.cl

${ }^{\dagger}$ Electronic address: leogrigorio@if.ufrj.br

${ }^{\ddagger}$ Electronic address: marcelosg@if.ufrj.br

$\S$ Electronic address: fmendez@usach.cl

๑Electronic address: clovis@if.ufrj.br 


\section{INTRODUCTION}

In this work we will construct a web of relations connecting known theories in $(2+1) D$ through duality and deformation. By deformation we mean a modification of the canonical structure of a theory. The deformation procedure, unlike duality, connects theories with different physical content. Therefore it is reasonable to suggest that the seemingly ad hoc deformations are in fact emulating some physical process. It is the ultimate purpose of this work to give a more precise meaning to this statement. Further, through the duality connection, we will be able to draw important conclusions concerning the duality of the physical processes themselves.

The deformation can be interpreted as defining a quantum theory with noncommutative fields as was introduced by [1, 2] and further studied by [3, 4, 5, 6]. This is a different set from the maybe more familiar spacetime noncommutativity, where the coordinates of spacetime are required to satisfy algebraic relations such as

$$
\left[x_{\mu}, x_{\nu}\right]=i \theta_{\mu \nu}
$$

with $\theta^{\mu \nu}$ a $(\text { lenght })^{2}$ parameter. In this way equation (1) defines a modification of the spacetime structure suppressing absolute spacetime localization by setting up a minimal area scale. In the noncommutative fields framework on the other hand, the modification is on the structure of the field phase space, for example, in $(3+1) D$ one would have

$$
\begin{array}{r}
{\left[\Phi_{i}(x), \Phi_{j}(y)\right]=i \varepsilon_{i j k} B^{k} \delta^{(3)}(x-y)} \\
{\left[\Phi_{i}(x), \Pi_{j}(y)\right]=i \delta_{i j} \delta^{(3)}(x-y)} \\
{\left[\Pi_{i}(x), \Pi_{j}(y)\right]=i \varepsilon_{i j k} \Theta^{k} \delta^{(3)}(x-y) .}
\end{array}
$$

Where $\Pi$ is the canonical momentum. The parameters $B$ and $\Theta$ appearing in (2) introduce new scales to the problem. They have canonical dimensions of length and mass, respectively, introducing an ultra-violet and an infra-red scale respectively if they are taken to be small. In $(3+1) D$ these deformations potentially break Lorentz and CPT invariance. As will be seen, in $(2+1) D$ example treated here they break $P$ and $T$.

The algebraic relations (2) are viewed as deformations of the canonical structure defining the relevant field theory. As such they lead to different theories describing distinct and new physical phenomena parameterized by the continuous deformation parameters. 
The widespread applications of these ideas rely on the fact that these deformations can be taken to lead to only tiny observed effects in known physical theories. Since there seems to be no inconsistences in their formulations they constitute a promising fertile ground for the study of new physics beyond presently known models. Therefore the rationale of the procedure deserves further inquire. The results discussed here can be seen also as a consistency check of the whole deformation procedure since it will be shown that it behaves appropriately under well known duality relations.

A great deal of work has been dedicated to the investigation of the physical, observable consequences of these controlled modifications in $(3+1) D$. The violation of Lorentz invariance following from these deformed structures leads to many interesting new phenomena. We may mention a few: in [1, 2] it was shown that, considering this deformation as modeling the high energy cosmic-ray radiation, it is possible to choose the deformation parameter such as to turn on or off the interaction of the cosmic-ray with the cosmic background radiation thus circumventing a GZK cutoff. In [7] it was shown that neutrino oscillations may be achieved even for massless neutrinos if we allow for Lorentz violation as defined by these deformations. In [8] baryogenesis was suggested as a possibility even in termal equilibrium, bypassing one of the Sakharov's criteria, if CPT is broken as it is by the deformation. Recently [9] inflationary scenarios were investigated in this context too, the deformation of the canonical structure is this case being defined not locally by a delta function as in (2) but in a region parameterized by a further adjustable parameter and the different scenarios were studied as a function of this parameter. This modified deformation was also discussed in [4].

These phenomenological consequences follow from the deformed induced models. It is this general interpretation of the deformation as a model generator that is of interest to us in the present work. In what follows we will stick with a particular example where all models related by duality and deformation are well known and studied. This will allow us to inquire not only about the consistency of the deformation procedure but also to obtain important new results concerning the relations between these models.

We will specifically address a $(2+1) D$ situation, a framework in which the deformation can be done without breaking the Lorentz symmetry but breaking $P$ and $T$ symmetries. We will demonstrate that the Self-dual (SD) model [10] can be obtained by a deformation of the canonical structure of the free scalar theory. This is a new result that displays the very interesting property of "rank jump", that is, the scalar theory is turned into a vector 
field theory after deformation. Incidentally this is the exact dual manifestation of the result discussed in [11]. There it was shown that a deformation of the canonical structure of the free Maxwell theory gives the Maxwell-Chern-Simons (MCS) theory [12] as a result (no "rank jump" here). Duality shows up in the fact that in $(2+1) D$ the free Maxwell theory has a dual description in terms of the free scalar theory and the MCS theory is well known to be dual to the SD theory [13].

With the result presented here we have then a neat picture connecting those four models through deformation and duality

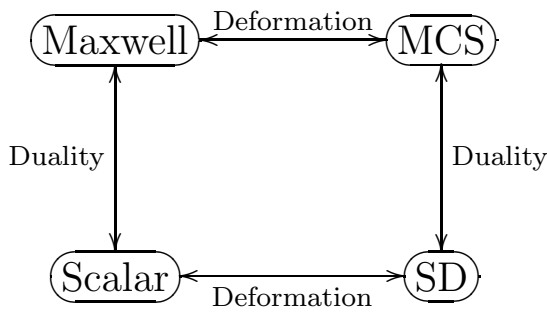

As already pointed out, another very important point which certainly deserves special attention is the possible physical interpretation of the deformation procedure. What it means, physically, an ordinary field theory to turn into a noncommutative fields theory? The upper link of the diagram (3), connecting the Maxwell theory with the MCS theory, has a well known physical interpretation: it describes the introduction of massive fermions in the theory which gives rise to vacuum polarization effects inducing the Chern-Simons term through radiative corrections. We think that one of the most important results of our work is to give a physical picture of what is going on in the lower link of the diagram, the scalar-SD connection. Because of the duality links, it can be interpreted as the dual phenomenon to the fermionic radiative corrections.

In the next section we will review the known connections in this picture: the Maxwellscalar duality and the MCS-SD duality, as well as the deformation of the Maxwell theory leading to the MCS theory. In the next following section we will present our result demonstrating the scalar-SD connection thus completing the picture (3) and establishing it as the dual manifestation of the Maxwell-MCS connection. We then proceed to a discussion of the physics underlying this picture which is the other result of this work. After that we finish with our concluding remarks. 


\section{DUALITY AND DEFORMATION}

The purpose of this section is to review some known results that are relevant to our work.

\section{A. Duality}

The two duality connections figuring in (3) share the same basic principles, it can be viewed in some sense as a change of variables. The duality procedure consists in doing a sequence of manipulations in the action making sure that none of them alter the physical content of the theory. Let us start with the Maxwell-Chern-Simons theory

$$
S_{M C S}=\int d^{3} x\left\{-\frac{1}{4} F_{\mu \nu} F^{\mu \nu}-\frac{m}{2} A_{\mu} \varepsilon^{\mu \nu \rho} \partial_{\nu} A_{\rho}\right\},
$$

where $F_{\mu \nu}=\partial_{\mu} A_{\nu}-\partial_{\nu} A_{\mu}$. It describes a topologically massive degree of freedom and formally reduces to the pure Maxwell theory if the topological mass $m \rightarrow 0$. Under an abelian gauge transformation $S_{M C S}$ changes by a surface term, so if it is defined in a suitable spacetime (topologically trivial and boundaryless) it is gauge invariant. But there is a remarkable property concerning this gauge symmetry structure: it is completely independent of the dynamical content of the theory. Thus when we talk about duality we are referring to dynamical duality. This is easily seen as follows (the following discussion is taken from [14] where a somewhat more complete assessment is made)

Consider the Lagrangian obtained from the MCS lagrangian by the introduction of an auxiliary field

$$
\begin{aligned}
\mathcal{L}_{M} & =\Pi_{\mu}\left(\varepsilon^{\mu \nu \rho} \partial_{\nu} A_{\rho}\right)+\frac{1}{2} \Pi_{\mu} \Pi^{\mu}-\frac{m}{2} A_{\mu} \varepsilon^{\mu \nu \rho} \partial_{\nu} A_{\rho} \\
& =\left(\Pi_{\mu}-\frac{m}{2} A_{\mu}\right)\left(\varepsilon^{\mu \nu \rho} \partial_{\nu} A_{\rho}\right)+\frac{1}{2} \Pi_{\mu} \Pi^{\mu}
\end{aligned}
$$

where $\Pi_{\mu}$ is an auxiliary vector-field which may be integrated out to give us back the MCS lagrangian. By making the redefinition $\Pi_{\mu}-\frac{m}{2} A_{\mu}=B_{\mu}$, we find

$$
\mathcal{L}_{M}=B_{\mu}\left(\varepsilon^{\mu \nu \rho} \partial_{\nu} A_{\rho}\right)+\frac{1}{2}\left(B_{\mu}+\frac{m}{2} A_{\mu}\right)\left(B^{\mu}+\frac{m}{2} A^{\mu}\right)
$$

Observe that by definition $B_{\mu}$ transforms as $B_{\mu} \rightarrow B_{\mu}-\frac{m}{2} \partial_{\mu} \Lambda$ whenever $A_{\mu} \rightarrow A_{\mu}+\partial_{\mu} \Lambda$, so that the gauge character of the $A_{\mu}$ field did not changed. Next we may perform a canonical 
transformation in the space of the fields to reveal the self-dual and pure gauge nature of the components

$$
\begin{aligned}
& B_{\mu}=\frac{1}{2}\left(A_{\mu}^{+}-A_{\mu}^{-}\right) \\
& A_{\mu}=\frac{1}{m}\left(A_{\mu}^{+}+A_{\mu}^{-}\right),
\end{aligned}
$$

which gives us

$$
\mathcal{L}_{M}=\frac{1}{2 m} A_{\mu}^{+} \varepsilon^{\mu \nu \rho} \partial_{\nu} A_{\rho}^{+}-\frac{1}{2 m} A_{\mu}^{-} \varepsilon^{\mu \nu \rho} \partial_{\nu} A_{\rho}^{-}+\frac{1}{2} A_{\mu}^{+} A^{+\mu},
$$

or, renaming $A_{\mu}^{+}=f_{\mu}$ and $A_{\mu}^{-}=A_{\mu}$

$$
\mathcal{L}_{M}=-\frac{1}{2 m} A_{\mu} \varepsilon^{\mu \nu \rho} \partial_{\nu} A_{\rho}+\frac{1}{2} f_{\mu} f^{\mu}+\frac{1}{2 m} f_{\mu} \varepsilon^{\mu \nu \rho} \partial_{\nu} f_{\rho}=\mathcal{L}_{C S}+\mathcal{L}_{S D},
$$

The first term is the standard Chern-Simons lagrangean and the remaining terms we recognize as the SD model [10]. It is clear by this procedure that the kind of gauge symmetry carried by the MCS theory have completely innocuous dynamical character as is well know by the properties of the pure Chern-Simons theory [15]. As we were able to separate this term we might say that the only information that the MCS theory has, which is not present in the SD model, regards the topological character of the space in which the theory is defined. It is very interesting and highly nontrivial that this separation is possible. It means, for example, that the energy propagating modes is given entirely by the SD sector in (9) but the energy eigenstates have a degeneracy parameterized by the Hilbert space of pure Chern-Simons theory (a topological degeneracy) [16]. In this way we establish the MCS-SD dynamical duality connection depicted in the diagram (3).

Incidentally eq.(4) describes the Maxwell theory if $m=0$. In this case, performing a partial integration, it is clear from (5) that $A_{\mu}$ appears as a Lagrange multiplier which enforces the constraint

$$
\varepsilon^{\mu \nu \rho} \partial_{\nu} \Pi_{\rho}=0 \Rightarrow \Pi_{\mu}=\partial_{\mu} \phi
$$

where $\phi$ is a scalar field. Substituting back in the action we obtain a free scalar theory, which proves the scalar-Maxwell duality connection as well.

\section{B. Deformation}

In this section we will reobtain the results of [11] regarding the connection between the free Maxwell theory and the MCS theory through the deformation of its canonical structure. 
The usual analysis gives rise to the following structure, consisting of the Hamiltonian,

$$
H=\int d^{2} x\left[\frac{1}{2} \vec{\Pi}^{2}+\frac{1}{2}(\nabla \times \vec{A})^{2}\right],
$$

the canonical brackets

$$
\begin{aligned}
& \left\{A_{i}(x), A_{j}(y)\right\}=0 \\
& \left\{A_{i}(x), \Pi^{j}(y)\right\}=\delta_{i}^{j} \delta^{(2)}(x-y) \\
& \left\{\Pi^{i}(x), \Pi^{j}(y)\right\}=0
\end{aligned}
$$

and the constraint

$$
\nabla \cdot \vec{\Pi}=0
$$

which could be incorporated in the definition of the Hamiltonian through a Lagrange multiplier which turns out to be the scalar potential $A^{0}$. The constraint reduces the phase space to a two dimensional manifold leaving the system with one degree of freedom as is known to be the case for the Maxwell theory in $(2+1) D$. The equations of motion, along with the constraint and the identifications $\vec{E}=\vec{\Pi}$ and $B=\nabla \times \vec{A}$ for the electric and magnetic fields respectively, gives us the Maxwell's equation in $(2+1) D$ (note that $B$ is a pseudo-scalar).

Now we turn to the deformation procedure. A deformation of the canonical brackets is an explicit modification of the dynamics of the theory. It is well known that in $(2+1) D$ a Chern-Simons (CS) term can be dynamically induced in the effective gauge theory by fermionic interactions. This can be inferred from symmetry considerations: if fermions are massive the effective gauge theory must break the discrete $P$ and $T$ symmetries since a fermionic mass does it in $(2+1) D$. But even if the fermions are massless the effective gauge theory breaks $P$ and $T$ because it is impossible to maintain gauge symmetry along with the $P$ and $T$ symmetries after regularization. So $P$ and $T$ are broken once we recognize that gauge symmetry, being just a redundancy in our description of the system, cannot disappear due to dynamical effects. We will take this recognition of the fundamental role of gauge symmetry as a guiding principle in obtaining the resulting theory after deformation.

As was done in [11] (see also [17]) we will impose the following deformation to the brackets 
(12)

$$
\begin{aligned}
& \left\{A_{i}(x), A_{j}(y)\right\}=0 \\
& \left\{A_{i}(x), \Pi^{j}(y)\right\}=\delta_{i}^{j} \delta^{(2)}(x-y) \\
& \left\{\Pi^{i}(x), \Pi^{j}(y)\right\}=\varepsilon^{i j} m \delta^{(2)}(x-y),
\end{aligned}
$$

where $m$, if taken small, is interpreted as an IR deformation (as it then defines a low energy scale for the deformation).

Alone, this deformation breaks the gauge invariance as the hamiltonian no longer has vanishing brackets with the original gauge generator

$$
G[\alpha]=\int d^{2} x \alpha(x) \nabla \cdot \vec{\Pi} .
$$

This prompt us to modify this generator defining a new constraint [17]

$$
\tilde{G}[\alpha]=\int d^{2} x \alpha(x) \partial_{i}\left(\Pi^{i}-\varepsilon^{i j} m A_{j}\right) .
$$

which may be seen to have vanishing brackets with the Hamiltonian (11). This new generator is obtained in a unique way just by requiring it to have vanishing brackets with the canonical momentum. To further construct the Lagrangian it is better to put the deformed brackets in a canonical form from which the symplectic structure can be immediately recognized. This is done by the following redefinition of the momenta

$$
\tilde{\Pi}^{i}=\Pi^{i}-\frac{\varepsilon^{i j} m}{2} A_{j}
$$

which cast the deformed brackets in the form of canonical ones

$$
\begin{aligned}
\left\{A_{i}(x), A_{j}(y)\right\} & =0 \\
\left\{A_{i}(x), \tilde{\Pi}^{j}(y)\right\} & =\delta_{i}^{j} \delta^{(2)}(x-y) \\
\left\{\tilde{\Pi}^{i}(x), \tilde{\Pi}^{j}(y)\right\} & =0 .
\end{aligned}
$$

The Lagrangian is then easily constructed if we take care of the new constraint also (here $\mathcal{H}$ is the Hamiltonian density corresponding to (11))

$$
\begin{aligned}
\mathcal{L} & =\sum_{i} \tilde{\Pi}^{i} \dot{A}_{i}-\mathcal{H}+A^{0} \partial_{i}\left(\tilde{\Pi}^{i}-\varepsilon^{i j} m A_{j}\right) \\
& =\sum_{i}\left(\Pi^{i} \dot{A}_{i}+\frac{m}{2} \varepsilon^{i j} A^{i} \dot{A}_{j}\right)-\frac{1}{2} \vec{\Pi}^{2}-\frac{1}{2}(\nabla \times \vec{A})^{2}+A^{0} \partial_{i}\left(\Pi^{i}-\varepsilon^{i j} m A_{j}\right) \\
& =-\frac{1}{4} F^{\mu \nu} F_{\mu \nu}-\frac{m}{2} \varepsilon^{\mu \nu \rho} A_{\mu} \partial_{\nu} A_{\rho}
\end{aligned}
$$


where the last equality is obtained from using the identification $\Pi^{i}=\dot{A}_{i}-\partial_{i} A^{0}$. This establishes the connection Maxwell/MCS through deformation as depicted in the diagram (3). Next we will complete the picture.

\section{THE FREE SCALAR / SELF-DUAL CONNECTION}

The picture depicted in (3) that have been drawn in the previous sections will be completed now. In this section we will establish the scalar/SD connection through deformation. This is to be viewed as the exact dual of the procedure discussed in the last section. But here the interesting property of "rank jump" will show up. The deformation considered here will turn a scalar field into a vector field. This is in tune with the scalar/Maxwell duality of course, but resulting from the deformation rather than of duality it may have a deeper meaning originating from new dynamical effects. These new effects would therefore be the exact dual of the radiative corrections emulated by the Maxwell/MCS connection discussed in the last section. We will have more to say about this in the concluding remarks.

Consider the free scalar theory defined by the Hamiltonian

$$
H=\int d^{2} x\left[\frac{1}{2} \Pi^{2}+\frac{1}{2}(\nabla \cdot \phi)^{2}\right]
$$

and the canonical brackets

$$
\begin{aligned}
\{\phi(x), \phi(y)\} & =0 \\
\{\phi(x), \Pi(y)\} & =\delta^{(2)}(x-y) \\
\{\Pi(x), \Pi(y)\} & =0 .
\end{aligned}
$$

We propose the following deformation to the brackets

$$
\begin{aligned}
\left\{\partial_{i} \phi(x), \partial_{j} \phi(y)\right\} & =m \varepsilon_{i j} \delta^{(2)}(x-y) \\
\{\phi(x), \Pi(y)\} & =\delta^{(2)}(x-y) \\
\{\Pi(x), \Pi(y)\} & =0 .
\end{aligned}
$$

and claim that the resulting system defined by $(20)$ and $(22)$ is equivalent to the SD system defined by the action:

$$
\mathcal{L}_{S D}=\frac{1}{2} f_{\mu} f^{\mu}+\frac{1}{2 m} f_{\mu} \varepsilon^{\mu \nu \rho} \partial_{\nu} f_{\rho} .
$$


In order to establish this link consider the equations of motion of the deformed scalar system

$$
\begin{aligned}
\dot{\Pi} & =\nabla^{2} \phi \\
\partial_{i} \dot{\phi} & =\partial_{i} \Pi-m \varepsilon_{i j} \partial^{j} \phi .
\end{aligned}
$$

These equations contain only derivatives of the scalar field. It must be further noticed that, if $m \neq 0$, these equations will have only solutions of the form $\phi=f(t)$ and $\Pi=c$, with $f$ a function of time only and $c$ a constant, both to be determined by initial conditions. This is then a trivial system with an homogeneous space energy density. But if we assign no meaning to the scalar field itself, claiming that its derivatives must be taken as the fundamental dynamical fields we are able to construct a non-trivial system. We will later elaborate a bit more on the possible physical phenomena behind this procedure, for now we will simply define the new dynamical variables for this system by the map

$$
\begin{aligned}
\Pi & =f^{0} \\
\partial^{i} \phi & =f^{i} .
\end{aligned}
$$

Equations (24), in terms of these new variables, can be cast in the form

$$
\begin{aligned}
\partial_{\mu} f^{\mu} & =0 \\
f^{i}+\frac{1}{m} \varepsilon^{i j} \partial_{j} f_{0}-\frac{1}{m} \varepsilon^{i j} \partial_{0} f_{j} & =0 .
\end{aligned}
$$

Observe further that by taking the derivative of the second equation in (26) and using the first we have

$$
\partial_{i} f^{i}=\frac{1}{m} \partial_{0}\left(\varepsilon^{i j} \partial_{i} f_{j}\right)=-\partial_{0} f^{0}
$$

Which allows us to conclude that

$$
f^{0}=-\frac{1}{m} \varepsilon^{i j} \partial_{i} f_{j}+C(x, y)
$$

That is, the field $f^{0}$ has its dynamics determined by the fields $f_{i}$ except for a time independent function, $C(x, y)$, which may be absorbed in a redefinition of $f^{0}$ without modifying the dynamics. By doing that we may compactly write the information contained in the above equations (26) and (28) in the form

$$
f^{\mu}+\frac{1}{m} \varepsilon^{\mu \nu \rho} \partial_{\nu} f_{\rho}=0
$$


which is recognized as the Euler-Lagrange equations for the $S D$ field whose action is defined by (23). This proves therefore that all non-trivial dynamical content of the deformed scalar field system is reproduced by the $S D$ system.

\section{THE PHYSICAL PICTURE}

In this section we will discuss the physical meaning of the connections just established in the previous sections. The diagram (3) we have constructed contain four links. The two duality relations are well known, as discussed in section II.A, so are their physical interpretation. They are a statement of the fact that the system admits an equivalent description in terms of another set of variables.

This equivalent description is much sought after in fully interacting theories where, by the general properties of duality, it would allow for a perturbative, weakly coupled, description of strongly interacting theories. The general implementation of duality in fully interacting theories is a remarkably difficult task though, with only very special examples known [18]. In our diagram, the duality links connects free theories but it can be readily generalized to include couplings with external sources. As we will see further ahead in this section, this is already sufficient to construct a picture of the physical mechanism behind the deformation.

The upper link connects the Maxwell theory with the MCS theory. It is known [19] that if massive fermions are taken to interact with the Maxwell field in $(2+1) D$ the effective description, after "integrating out" the fermions, is the MCS theory to lowest order in the inverse fermion mass. This is an excellent approximation for the system if the fermions are heavy. The induced CS term has a nice physical interpretation. If the fermions are much heavier than the other relevant particles in the system they cannot be excited as real particles, their dynamics are frozen, but they will contribute quantum mechanically through fermionic loops which will surely disturb the propagation of the lighter particles. But massive fermions have very peculiar properties in $(2+1) D$ which is intimately related to the nature of spin in this dimensionality. In $(2+1) D$ a Dirac fermion has only two components as follows from the representation of the Dirac matrices in terms of the $2 \times 2$ Pauli matrices. Therefore electrons have a definite spin component, they will "point up or down", and this is defined by the sign of the mass term in the action, which properly violates $\mathrm{P}$ and $\mathrm{T}$ symmetries. As a consequence it follows that the fermionic quantum condensate in 
which the lighter particles propagate is a $P$ and $T$ symmetry breaking state and the effective description of the electromagnetic propagation must include a term with this information, that is, the CS term.

In fact, the CS term may be viewed as an effective interaction (representing the self energy of the fermion; its mass term) of the electromagnetic current coupled with an induced current. Explicitly:

$$
<M \bar{\psi} \psi>=<J^{\mu}>A_{\mu}
$$

where the induced current takes the form

$$
<J^{\mu}>=\frac{e^{2}}{4 \pi} \frac{M}{|M|} * F^{\mu}
$$

Except for the $4 \pi$ factor, this expression can be obtained only on the grounds of dimensional analysis. The preceding discussion of the fermionic condensate is essentially a reproduction of remarks found in [19] and [20]. It contains the essential ingredients for an interpretation of the dual formulation.

The question is: how this physical picture presents itself in the dual formulation? In the original picture just discussed we may think of the deformation as describing a condensation process of sources minimally coupled with the electromagnetic field. Consider the Maxwell field minimally coupled with an external conserved current

$$
\mathcal{L}_{\text {Max }}=-\frac{1}{4} F^{\mu \nu} F_{\mu \nu}-e J_{\mu} A^{\mu},
$$

and the conserved current can be written as

$$
J^{\mu}=\varepsilon^{\mu \nu \rho} \partial_{\nu} K_{\rho} .
$$

In doing this we are introducing a symmetry in the theory which accounts for the freedom that we have in choosing the brane $K_{\mu}$

$$
K_{\mu} \rightarrow K_{\mu}+\partial_{\mu} \chi
$$

In this formulation $J^{\mu}$ describes a delta-like current which borders the surface traced by the brane $K_{\mu}$. The condensation process described previously thus can be seen as the phenomenon of the singular brane $K_{\mu}$ turning into a field which in a first approximation (in a momentum expansion) is identified with the gauge field $A_{\mu}$. 
A dual representation of (32) is constructed following the same path traced in section II.A. The result is the scalar field non-minimally coupled with the brane $K_{\mu}$

$$
\mathcal{L}_{S c}=\frac{1}{2}\left(\partial_{\mu} \phi-e K_{\mu}\right)^{2}
$$

The brane symmetry is realized here taking into account the compactness of the scalar field

$$
\begin{aligned}
K_{\mu} & \rightarrow K_{\mu}+\partial_{\mu} \chi \\
\phi & \rightarrow \phi-e \chi .
\end{aligned}
$$

Thus we see that the minimally coupled sources of the original formulation presents themselves as defects in the dual formulation. Therefore the physical picture in the dual formulation is a defect condensation.

To proceed further we refer to a very useful procedure to deal with this phenomenon in this formulation, it is called the Julia-Toulouse mechanism. It was further investigated and extended to deal with general relativistic p-form theories in the presence of defects in a seminal work by Quevedo and Trugenberger [21]. The prescription they have proposed was able to deliver a very interesting picture of the dual phenomenon to the Higgs mechanism and they were also able to draw important results concerning the puzzle of the axion mass.

The Julia-Toulouse prescription (or mechanism) amounts to the construction of a field theory in which the defects are condensed. The details about the phenomenon that drives the condensation are not addressed nor they need to be because with very general assumptions an unique form of the theory after the condensation may be constructed. Quevedo and Trugenberger considered as the only assumptions that the resulting effective theory was renormalizable, Lorentz invariant, respects all the symmetries of the problem and be constructed as a derivative expansion with respect to the new scale defined by the characteristic density of the condensate.

In the present situation we are searching for a theory describing the physics of a $P$ and $T$ symmetry breaking condensate. Taking as the starting point the scalar field action (35) we follow the Julia-Toulouse prescription by first identifying the Stuckelberg-like brane symmetry (36) invariant of the theory. This is obviously given by:

$$
f_{\mu}=\partial_{\mu} \phi-e K_{\mu}
$$

The condensation process is a proliferation of the defects. A defect means a singularity in the scalar field, that is, the scalar field is not well defined at the position of a defect. As 
the condensation process becomes energetically favored the scalar field becomes more and more singular until it is not defined anywhere and only the brane invariant field $f_{\mu}$ (37) retains any physical meaning. It describes the excitation field of the condensate. The JuliaToulouse prescription prompt us to add terms to the lagrangian to account for the dynamics of these excitations. The first such term in a derivative expansion that breaks the $P$ and $T$ symmetries is the CS term. So we arrive at the following effective description of the system after condensation of defects takes place

$$
\mathcal{L}_{S D}=\frac{1}{2} f_{\mu} f^{\mu}+\frac{1}{2 m} f_{\mu} \varepsilon^{\mu \nu \rho} \partial_{\nu} f_{\rho}
$$

where $m$ is interpreted as the density on the condensate.

We think that this is an important result. It gives not only a beautiful physical picture of the otherwise ad hoc deformation procedure in this instance, but also constitutes a straightforward generalized application of the Julia-Toulouse mechanism. Observe that this mechanism is here a dual representation for the fermionic radiative processes, this suggests that there is more to the Julia-Toulouse mechanism than originally thought.

\section{CONCLUSIONS AND PERSPECTIVES}

Besides providing a new representation of the well known $S D$ system through scalar fields with deformed brackets, a nontrivial result by itself, the results described in this work provides a broad perspective on the physical significance of the deformation procedure. It was already suggested that the physical mechanism behind such deformations is associated with radiative corrections [17], and the deformation would be an emulation of such processes. A well known deformation with this property is the Maxwell/MCS deformation. The dual deformation obtained here, the scalar/SD deformation, must thus be the emulation of a dual physical phenomenon. A remarkable property of this new deformation is the phenomenon of "rank jump", that is, the change of the tensorial nature of the fundamental dynamical fields in the theory. Using the fact that the phenomenon of "rank jump" may sometimes be associated with the condensation of topological defects, a process better described by

the so called Julia-Toulouse mechanism in the modern form discussed by [21], we were able to draw a physical picture of the deformation procedure in this $(2+1) D$ setting. An interesting conclusion is the dual representation of the radiative processes. The known 
image of the vacuum as a medium with quantum fluctuations of heavy particles modifying the propagation of lighter ones is reinterpreted as a condensate of defects in which the dual of the lighter fields propagates.

\section{ACKNOWLEDGMENTS}

Some of us, LG, MSG and CW would like to thank Fundação de Amparo à Pesquisa do Estado do Rio de Janeiro (FAPERJ) and Conselho Nacional de Desenvolvimento Científico e Tecnológico (CNPq) and CAPES (Brazilian agencies) for financial support. JG and FM were partially supported by FONDECYT-Chile grants 1050114, 1060079. CW and MSG would like to thank Departamento de Física, Universidad de Santiago de Chile for kind hospitality and support.

[1] J. M. Carmona, J. L. Cortes, J. Gamboa and F. Mendez, Phys. Lett. B 565 (2003) 222 arXiv:hep-th/0207158.

[2] J. M. Carmona, J. L. Cortes, J. Gamboa and F. Mendez, JHEP 0303 (2003) 058 arXiv:hep-th/0301248.

[3] G. Mandanici and A. Marciano, JHEP 0409 (2004) 040 arXiv:hep-th/0406197.

[4] A. P. Balachandran, A. R. Queiroz, A. M. Marques and P. Teotonio-Sobrinho, arXiv:0706.0021 [hep-th].

[5] A. K. Das, J. Gamboa, J. Lopez-Sarrion and F. A. Schaposnik, Phys. Rev. D 72 (2005) 107702 arXiv:hep-th/0510002.

[6] J. Gamboa, J. Lopez-Sarrion and A. P. Polychronakos, Phys. Lett. B 634 (2006) 471 arXiv:hep-ph/0510113.

[7] P. Arias, J. Gamboa, J. Lopez-Sarrion, F. Mendez and A. K. Das, Phys. Lett. B 650 (2007) 401 arXiv:hep-ph/0608007.

[8] J. M. Carmona, J. L. Cortes, A. K. Das, J. Gamboa and F. Mendez, Mod. Phys. Lett. A 21 (2006) 883 arXiv:hep-th/0410143.

[9] L. Barosi, F. A. Brito and A. R. Queiroz, JCAP 0804 (2008) 005 [arXiv:0801.0810 [hep-th]].

[10] P. K. Townsend, K. Pilch and P. van Nieuwenhuizen, Phys. Lett. B 136 (1984) 38. 
[11] J. R. Nascimento, A. Y. Petrov and R. F. Ribeiro, Europhys. Lett. 77, 51001 (2007) arXiv:hep-th/0601077].

[12] S. Deser, R. Jackiw and S. Templeton, Annals Phys. 140, 372 (1982) [Erratum-ibid. 185, 406.1988 APNYA,281,409 (1988 APNYA,281,409-449.2000)].

[13] S. Deser and R. Jackiw, Phys. Lett. B 139 (1984) 2366.

[14] M. S. Guimaraes, L. Grigorio and C. Wotzasek, "The dual of the Carroll-Field-Jackiw model," arXiv:hep-th/0609215.

[15] G. V. Dunne, "Aspects of Chern-Simons theory," arXiv:hep-th/9902115.

[16] M. Asorey, F. Falceto and S. Carlip, Phys. Lett. B 312, 477 (1993) arXiv:hep-th/9304081.

[17] J. Gamboa and J. Lopez-Sarrion, Phys. Rev. D 71, 067702 (2005) arXiv:hep-th/0501034.

[18] L. Alvarez-Gaume and F. Zamora, "Duality in quantum field theory (and string theory)," arXiv:hep-th/9709180.

[19] A. N. Redlich, Phys. Rev. D 29, 2366 (1984).

[20] F. Wilczek, "Fractional statistics and anyon superconductivity," Singapore, Singapore: World Scientific (1990).

[21] F. Quevedo and C. A. Trugenberger, Nucl. Phys. B 501, 143 (1997) arXiv:hep-th/9604196. 